\title{
La iluminación en el videojuego. Aproximación a las claves que guían las interacciones del jugador en los entornos tridimensionales lúdicos.
}

\author{
The lighting in the game. \\ Approach to the keys that guide player \\ interactions on three-dimensional ludic \\ environments.
}

Marta Fernández Ruiz

Doctora en Investigación en Medios de Comunicación (Universidad Carlos III)

Fecha de recepción: 19 de octubre de 2013

Fecha de revisión: 19 de diciembre de 2013

Para citar este artículo: Fernández Ruiz, M. (2014): La iluminación en el videojuego. Aproximación a las claves que guían las interacciones del jugador en los entornos tridimensionales lúdicos, Icono 14, volumen (12), pp. 293-318. doi: 10.7195/ri14.v12i1.650 


\section{Resumen}

Si bien los videojuegos actuales se caracterizan en su mayoría por su gran riqueza visual (texturas en alta definición, iluminación dinámica, etc), ésta no siempre tiene un fin meramente estético o decorativo, sino que puede adoptar un papel funcional al guiar visualmente al jugador en las diferentes interacciones que debe realizar en el juego. El propósito de este trabajo es explorar el papel la iluminación como recurso expresivo empleado para condicionar los movimientos y las acciones del jugador en los videojuegos. De acuerdo a ello, se ha desarrollado un análisis de contenido sobre una muestra de videojuegos comerciales actuales. El trabajo concluye con una descripción de las principales propiedades de la luz empleadas en el videojuego como forma de facilitar al jugador el acceso interactivo en los entornos tridimensionales.

\section{Palabras clave}

Iluminación - Interacción - Videojuegos - Diseño - Interfaz - Comunicación

\section{Abstract}

Although one of the main features of current video games is their visual richness (high definition textures, dynamic illumination, etc), not only this richness is used for decorative or aesthetic purposes, but it can also have a functional aim, namely guiding users' interactions inside the game environments. The goal of this research is to assess the role of lighting as a visual expressive resource that is used to guide the players' movements and interactions. According to this, a content analysis on a sample of current commercial video games has been carried out. The paper concludes with a description of the main light properties that are used in video games as a way to invite the user to interaction inside three-dimensional spaces.

\section{Key Words}

Lighting - Interaction - Video games - Design - Interface - Communication 


\section{Introducción}

Los avances tecnológicos han dado lugar a un gran realismo gráfico y a mayores oportunidades de interacción en los entornos de los videojuegos, lo que ha llevado a proporcionar una mayor sensación de presencia y de libre albedrío en los usuarios. No obstante, los entornos de gran parte de los videojuegos se representan a partir de recursos expresivos (mapas, brújulas, puntos de vista...) que tienden a mostrar al jugador los items con los que éste puede interaccionar, así como a restringir el acceso a aquellos elementos que no van a ayudar al jugador a progresar en su recorrido interactivo.

Una de las heurísticas de usabilidad en los videojuegos consiste en que la representación audiovisual sirva de apoyo a las interacciones que tienen lugar en el juego (Isbister \& Schaffer, 2008); es decir, la capa sonora, háptica y visual del videojuego debe mostrar al jugador la información y el feedback de una manera atractiva y fácil de entender. Estudios anteriores han tratado el papel de diferentes dispositivos visuales en el videojuego (especialmente el de la cámara virtual) y su papel a la hora de facilitar y promover las interacciones del jugador en los entornos tridimensionales de los videojuegos (Nitsche, 2008). No obstante, recursos expresivos como la iluminación o el color han recibido escasa atención por parte de la comunidad académica. La iluminación, como componente esencial de los niveles de los videojuegos, parece ejercer un papel determinante, no sólo a nivel narrativo o evocativo, sino también para dirigir la atención del jugador hacia aquellos caminos, personajes y objetos que le van a permitir progresar en el juego. En medios como la pintura, la fotografía o el cine la iluminación ha tendido a guiar la atención selectivamente, de conformidad con el significado pretendido (Arnheim, 1995). Si llevamos esta idea a los entornos virtuales tridimensionales lúdicos, el objetivo principal de la iluminación sería el de dirigir la atención del jugador hacia las áreas de interés, lo que en los videojuegos se expresa a través de la navegación y la interacción. Un conocimiento amplio del lenguaje del videojuego puede contribuir a enriquecer el diseño de niveles, al promover la comunicación entre el diseñador y el usuario a través de los espacios y escenarios del juego, y al permitir optimizar los recursos empleados en los procesos de renderizado. A día de hoy se considera que la labor del diseñador 
de niveles, más allá de establecer los principales obstáculos y desafíos, así como las diferentes atmósferas, consiste en crear experiencias de juego a partir de la interacción del jugador con el espacio lúdico (Canossa, 2006). La experiencia de juego ha sido definida como el conjunto de sensaciones, pensamientos, sentimientos, acciones y significados que experimenta el jugador cuando entra en contacto con el videojuego (Ermi \& Mäyrä, 2005).

En este contexto, este trabajo pretende observar, describir y analizar el papel de la iluminación en los entornos tridimensionales de los videojuegos como herramienta para configurar experiencias de juego. En concreto, su papel para guiar y promover la navegación y la interacción del usuario y, por tanto, para contribuir a que la experiencia del usuario sea fluida (al evitar tiempos muertos derivados de la búsqueda excesiva de los elementos con los que es necesario interactuar o de los espacios por los que hay que continuar el recorrido interactivo) y significativa (en la medida en que sugieren al jugador la realización de una serie de acciones que tendrán una repercusión en el curso del juego).

En el ámbito académico del videojuego la iluminación ha sido estudiada principalmente a partir de los comportamientos del usuario empírico y especialmente en lo relativo a las emociones que determinadas condiciones lumínicas provocan en el jugador y las consecuencias que ello tiene en el desarrollo de las actividades dentro de los entornos tridimensionales lúdicos (Knez \& Niedenthal, 2008). También se han realizado análisis de contenido de videojuegos existentes, pero desde la perspectiva del potencial evocativo de la iluminación en videojuegos de un género determinado: el survival horror (Niedenthal, 2008).

Por otra parte, diferentes estudios sobre la atención visual en videojuegos tridimensionales han revelado que la atención visual del jugador, al igual que en medios como el teatro o el cine, se centra en objetos que destacan visualmente con respecto a los que se encuentran a su alrededor. Sin embargo, el estudio no hace alusión a la iluminación, sino al color de las superficies de los objetos y al movimiento de los mismos (Seif El-Nasr \& Yan, 2006).

También se han llevado a cabo estudios sobre la iluminación y su capacidad 
de captar la atención visual del jugador. En este contexto se ha desarrollado un prototipo para que, a tiempo real, la iluminación ayude a que la mirada del jugador se dirija hacia las zonas importantes del espacio interactivo. Se trata de un estudio del comportamiento del jugador, centrado en comparar la actuación del jugador en entornos con iluminación estática y en entornos con iluminación dinámica (a diferencia de la iluminación estática, que es pre-calculada por el ordenador en el momento de la creación del juego, la iluminación dinámica se calcula constantemente durante la ejecución del juego en función de las diferentes interacciones que realiza el jugador sobre el entorno), partiendo de la hipótesis de que la iluminación dinámica favorece la fijación de la atención visual del jugador sobre elementos importantes. Si bien se hace referencia a que dicho prototipo permite centrar la atención visual del jugador hacia zonas importantes, no hacen mención al papel explícito de la iluminación para guiar las interacciones y la navegación del jugador (Seif El-Nasr, Vasilakos \& Zupko, 2009).

Finalmente, se han encontrado estudios sobre los patrones de diseño que se emplean para guiar el movimiento del jugador en videojuegos tridimensionales (Milam \& Seif El-Nasr, 2010a). En ninguno de estos patrones se aborda en profundidad la iluminación.

Se considera, por tanto, que no se ha realizado un estudio académico, desde la disciplina de la comunicación, que aborde las diferentes formas en las que se aplica la iluminación con el fin de guiar la navegación y las interacciones del jugador. Se pasa a continuación a tratar con más detalle estas cuestiones.

\subsection{Play personas}

Hablar de iluminación e interacción en los videojuegos implica hablar del empleo de este elemento compositivo como una estrategia del diseñador para que el jugador realice una serie de actividades que le lleven a una experiencia similar a la que el diseñador había previsto. Si bien siempre hay riesgos de que los jugadores no se comporten como había pronosticado el diseñador (juego emergente), es posible, mediante determinadas estrategias, hacer que determi-

DOI: ri14.v12i1.650 | ISSN: 1697-8293 | Año 2014 Volumen 12 N 1 | ICONO14 
nados comportamientos sean más probables que otros. El término persona hace referencia a arquetipos detallados de usuarios que representan diferentes grupos de comportamientos, actitudes, aptitudes, metas y motivaciones observadas e identificadas en la fase de investigación del diseño de un producto (Cooper, Reimann \& Cronin, 2007). En el ámbito específico del videojuego, la noción de play-persona se ha empleado para designar los diferentes patrones de comportamiento o estilos de juego que los jugadores desempeñan. Estos patrones emergen del conjunto de posibilidades de interacción y navegación que las reglas del juego y los espacios permiten.

De las diferentes acepciones existentes de este término (Tychsen \& Canossa, 2008), la más cercana a la investigación presente es la que establece que las play personas consisten en una presuposición del diseñador, durante el proceso de creación del videojuego, sobre el comportamiento y los estilos de juego de los jugadores. Este trabajo pretende observar si la iluminación es uno de los elementos visuales expresivos a través de los cuales el diseñador plasma su idea de jugador modelo.

\subsection{La atención visual en el videojuego}

La atención visual en el videojuego ha sido tratada como un proceso cognitivo y perceptual consistente en centrar la atención en un determinado aspecto del entorno, mientras se ignoran otros (Sennersten, 2008). El estudio de este proceso ha partido del supuesto de que existen dos mecanismos de control sobre los que la atención visual se desarrolla. El primero de ellos es conocido como procesamiento bottom-up o proceso de pre-atención dependiente de la prominencia de los objetos (prominencia que se consigue haciendo destacar el objeto sobre el fondo) e independiente de la tarea que el sujeto realiza. El segundo es conocido como procesamiento top-down, mucho más lento que el anterior, controlado por la voluntad y por tanto, dependiente de la tarea específica en ejecución. Mediante un experimento consistente en observar los patrones de atención visual registrados mediante sistemas de eye tracking, Seif El-Nasr y Yan (2006) pudieron confirmar que tanto el procesamiento bottom-up como el topdown se dan en el videojuego tridimensional, si bien es éste último el que mayor

ICONO14 | Año 2014 Volumen $12 N^{\circ} 1$ | ISSN: 1697-8293 | DOI: ri14.v12i1.650 
influencia ejerce en el desarrollo de la atención visual. La investigación sobre la atención visual en los videojuegos puede enriquecer el diseño de los entornos lúdicos y dar lugar a experiencias de juego más fluidas y satisfactorias, ya que el establecimiento de focos de atención puede ayudar a reducir la frustración del jugador en los momentos en los que no puede identificar visualmente los elementos clave para el juego.

\subsection{Iluminación y movimiento en entornos arquitectónicos}

No han sido pocos los estudios que asemejan determinados aspectos formales del videojuego con soluciones visuales ya adoptadas en el cine. No obstante, estudios recientes establecen una mayor similitud entre el videojuego y la arquitectura (Niedenthal, 2008). La arquitectura se diferencia de otras artes visuales principalmente porque es experimentada mediante el movimiento físico a través y alrededor de la misma (Michel, 1996).

El movimiento humano alrededor de la arquitectura difiere en función de si el entorno es conocido o no. En un territorio familiar, los caminantes se mueven de una manera habitual y apenas prestan atención al entorno en el que se encuentran. Pero cuando un edificio no es familiar, se mueven por el mismo de una manera exploratoria, mirando en todas las direcciones, dudando y a veces retrocediendo algunos pasos (Bechtel, 1967). Es en estos sitios donde la percepción espacial de la arquitectura es altamente significativa. La tarea del diseñador de entornos en estos casos es minimizar la confusión mientras los viandantes buscan su camino, al mismo tiempo que establece determinados puntos de interés a lo largo de estos caminos (Michel, 1996).

En este contexto, la iluminación ejerce una influencia poderosa en el observador debido principalmente a lo que se conoce como fototropismo. Si bien este término hace referencia al crecimiento de las plantas hacia la fuente de luz, se ha obtenido evidencia de que esta tendencia también se da en los seres humanos. Ante dos caminos con intensidades lumínicas diferentes, las personas tienden a elegir el camino de mayor intensidad lumínica (Taylor \& Socov, 1974). 


\section{Metodología}

Para llevar a cabo el análisis de la iluminación en los videojuegos se ha realizado un análisis de contenido cualitativo y cuantitativo. La parte cualitativa ha consistido en la observación y la anotación de datos significativos con el fin de ofrecer una descripción detallada del uso de la iluminación como recurso expresivo para guiar la navegación y las interacciones del jugador. Asimismo se han empleado determinadas herramientas con las que medir algunos parámetros de la luz, principalmente a partir del software Adobe Photoshop. El análisis cuantitativo se ha llevado a cabo con el fin de determinar la frecuencia a la que se producen determinados usos de la iluminación, para lo cual se han realizado operaciones estadísticas simples (porcentajes).

Antes de proceder al análisis se ha realizado un pre-análisis consistente en la constitución de un corpus, en la toma de contacto con los videojuegos pertenecientes a dicho corpus y en el establecimiento de un sistema de unidades de registro y categorías.

En este sentido se ha procedido a realizar sesiones de juego con una muestra determinada de videojuegos a una profundidad de un tercio del número total de los niveles de cada uno de estos videojuegos (Tabla 1). La elección de dicha muestra ha obedecido a cinco criterios.

El primero de ellos ha sido la aceptación y circulación social. Para conocer los videojuegos más populares entre la crítica y los jugadores, se han tenido en cuenta, por una parte, las valoraciones de la crítica sobre los videojuegos lanzados al mercado y, por otra, la posición de dichos juegos en listas de ventas. Para conocer las apreciaciones de la crítica se han consultado los rankings de los videojuegos mejor puntuados en los años 2010 y 2011 y lanzados al mercado para las plataformas Play Station 3 y Xbox 360 en el sitio web Metacritic ${ }^{1}$, un agregador que recoge las puntuaciones que sitios web, periódicos y revistas dan de los videojuegos (así como de otros productos culturales) para crear una puntuación media. De estos rankings, se han escogido aquellos que han tenido una puntuación igual o superior a 75 (la máxima es de 100). 
También se ha limitado la búsqueda de juegos a los que se han lanzado para las plataformas Xbox 360 y PlayStation 3. Éstas fueron las primeras consolas con gráficos en alta definición. Los títulos de éstas se caracterizan, generalmente, por un mayor aprovechamiento de las posibilidades que la tecnología permite en la creación de gráficos por ordenador.

Asimismo, se han buscado juegos actuales. El análisis de videojuegos recientes desarrollados por los nuevos motores de juego (Unreal, Cry Engine, etc.) permite un acercamiento a las últimas tendencias en lo relativo al empleo de gráficos para la interacción. Por ello la muestra comprende videojuegos que han sido lanzados al mercado entre los años 2010 y 2011.

Por otra parte, la investigación presente trata de limitarse a los juegos de progresión, frente a los de emergencia. Si bien los juegos de emergencia se caracterizan por depender de un limitado número de reglas cuya combinación da lugar a grandes variaciones en los acontecimientos del juego (como ocurre, por ejemplo, en los juegos de cartas y de mesa, así como en casi todos los juegos de estrategia y de rol), los de progresión se caracterizan por ofrecer una secuencia de eventos prefijada. El jugador debe realizar un conjunto de acciones en un orden determinado para completar el juego (Juul, 2002). Esta estructura nace con el videojuego, a partir, principalmente, del género de aventura. En el panorama comercial actual, los juegos de progresión se corresponden generalmente con los géneros de acción-aventura, first person shooters y plataformas, y estos son los géneros de los que se compone la muestra en este trabajo de investigación.

Finalmente, se ha restringido el análisis a videojuegos tridimensionales. A diferencia de algunos videojuegos bidimensionales como Space Invaders (Taito, 1977) o Pac-Man (Namco, 1980), donde el jugador tiene una visión completa de lo que ocurre en el juego, los videojuegos tridimensionales esconden elementos de la vista de los jugadores, al tiempo que permiten una navegación más compleja (ejes $\mathrm{x}, \mathrm{y}, \mathrm{z}$ ). Ello motiva una mayor presencia de ayudas visuales a la navegación y la interacción en este tipo de videojuegos. 


\begin{tabular}{|l|c|l|l|}
\hline \multicolumn{1}{|c|}{ Título } & Año de lanzamiento & \multicolumn{1}{c|}{ Publisher } & \multicolumn{1}{c|}{ Desarrolladora } \\
\hline Alan Wake & 2010 & $\begin{array}{l}\text { Microsoft Game Stu- } \\
\text { dios }\end{array}$ & Remedy Entertainment \\
\hline Bioshock 2 & 2010 & 2K Games & 2K Games \\
\hline $\begin{array}{l}\text { Castlevania. Lords of } \\
\text { Shadow }\end{array}$ & 2010 & Konami & Mercury Steam \\
\hline Crysis 2 & 2011 & Electronic Arts & Crytek \\
\hline Dante's Inferno & 2010 & Electronic Arts & Visceral Games \\
\hline Darksiders & 2010 & THQ & Vigil Games \\
\hline Dead Space 2 & 2011 & Electronic Arts & Visceral Games \\
\hline $\begin{array}{l}\text { Enslaved. 0dyssey to } \\
\text { the West }\end{array}$ & 2010 & Namco Bandai Games & Ninja Theory \\
\hline God of War 3 & 2010 & $\begin{array}{l}\text { Sony Computer Enter- } \\
\text { tainment }\end{array}$ & SCE Studios \\
\hline $\begin{array}{l}\text { Shadows of the Dam- } \\
\text { ned }\end{array}$ & 2011 & Electronic Arts & $\begin{array}{l}\text { Grasshopper Manufac- } \\
\text { ture }\end{array}$ \\
\hline Singularity & 2010 & Activision & Raven Software \\
\hline Uncharted 3 & 2011 & $\begin{array}{l}\text { Sony Computer Enter- } \\
\text { tainment }\end{array}$ & Naughty Dog \\
\hline
\end{tabular}

Tabla 1. Muestra de videojuegos sometidos a análisis. Fuente: Elaboración propia.

Tras ello, se ha procedido a la obtención de vídeos de las sesiones de juego o walkthroughs que la comunidad de jugadores comparte en sitios como YouTube. Dichos vídeos se corresponden con los niveles que previamente ha jugado la autora de este trabajo. El material que la comunidad de jugadores comparte en Internet ha sido un recurso empleado por otros investigadores (Milam \& Seif El-Nasir, 2010b; Canossa, 2006). Con este tipo de material es posible obtener una información más amplia que la que se podría obtener si sólo se hubiera procedido a la captura de las sesiones de juego de la investigadora, dado que quienes comparten vídeos de sus secuencias de juego en Internet suelen ser jugadores experimentados que lo hacen a modo de guía.

Una vez obtenido el material sobre el que se realiza el análisis, se ha procedido a la codificación, entendida ésta como el proceso de creación de unidades de registro y la elección de categorías. 


\subsection{Unidades de Registro}

La unidad de registro es definida como el segmento de contenido que será necesario considerar como unidad de base con miras a la categorización y al recuento frecuencial (Bardin, 1996). Para esta investigación, se han establecido como unidades de registro las ocasiones en las que la iluminación, a partir de diferentes propiedades de la luz, es empleada para llamar la atención visual del jugador hacia un objeto o non-player character (personaje no controlado por el jugador) para mostrar que éste es interactivo, o hacia un camino (una puerta, una ventana, etc.) para mostrar que el jugador debe dirigir a su avatar hacia la dirección que indica la luz (Figura 1).

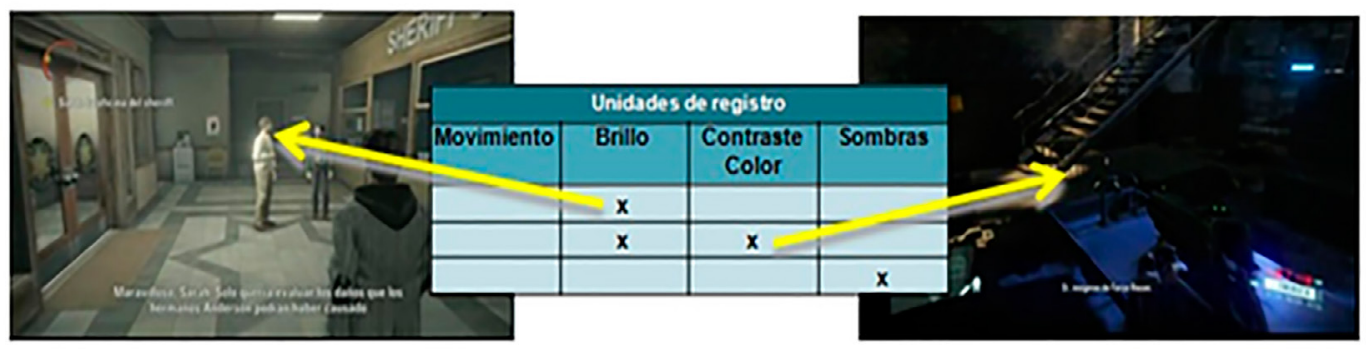

Figura 1: Ejemplo de tabla sobre la que se han registrado las diferentes propiedades de la luz empleadas para guiar la navegación o las interacciones del jugador.

Tras la toma de contacto con los videojuegos pertenecientes a la muestra, se ha determinado que las propiedades de la luz susceptibles de formar parte de una unidad de registro son el movimiento, la intensidad lumínica, el contraste cromático y las sombras.

El movimiento es la incitación visual más fuerte a la atención (Arnheim, 1995). El movimiento rápido de una persona, o de un objeto que se cae, llamarán inmediatamente la atención del ojo humano, incluso aunque el estímulo se produzca en una zona periférica del campo de visión. Cuando el movimiento se combina con una intensidad lumínica elevada, se produce una atracción excepcionalmente poderosa para el ojo. Las luces intermitentes o en movimiento garantizan que la mirada se dirija hacia ellas (Michel, 1996).

DOI: ri14.v12i1.650 | ISSN: 1697-8293 | Año 2014 Volumen 12 No 1 | ICONO14 
La intensidad lumínica, más conocida como brillo, es la impresión subjetiva de la luz cuando ésta ilumina una superficie y se compara con otra (Michel, 1996). Esta definición pone de relieve la relatividad del brillo, al considerar que éste no es un parámetro aislado, sino que los observadores necesitan experimentar dos superficies simultáneamente para juzgar si una de ellas es más brillante que otra. Cualquier parte de un entorno llamará la atención del observador si es iluminada con una elevada intensidad lumínica. No es casual que el brillo en los entornos tridimensionales de los videojuegos suela ser elevado en áreas que permiten el acceso del jugador a nuevos espacios que explorar, como las puertas.

Para la observación de la intensidad lumínica, y con el fin de obtener información más precisa y certera, se ha empleado la herramienta Umbral del programa Adobe Photoshop. Investigaciones previas han empleado el Histograma del citado software para conocer las condiciones de iluminación (Niedenthal, 2008) y la paleta de color aplicada al nivel de un videojuego (Canossa, 2006). El Histograma proporciona información sobre cuántos píxeles de la imagen tienen un color (rojo, azul o verde) o un nivel de luminosidad (de entre las 256 líneas de la escala de grises). No obstante, el Histograma no contiene información espacial de la imagen. Si bien indica qué nivel medio de luminosidad hay en una imagen, o cuál es el color dominante, no indica los lugares específicos de la imagen en los que están localizados.

Dado que este trabajo pretende observar el brillo de determinadas áreas en comparación con la de otras en una misma imagen, se ha optado por buscar herramientas similares que obedezcan más directamente a los propósitos y necesidades de esta investigación. Por ello, para identificar el área de mayor intensidad lumínica se ha empleado la herramienta Umbral, que determina las áreas más claras y más oscuras de una imagen. Mediante esta herramienta, todos los píxeles más claros que el umbral se muestran en blanco (Figura 2); mientras que todos los píxeles más oscuros se representan en negro.

Durante la realización del análisis, las ocasiones en las que la zona de mayor intensidad lumínica coincidía con el área en la que se encontraba el objeto, personaje o camino importante para el progreso en el juego eran recogidas en una tabla de análisis.

ICONO14 | Año 2014 Volumen $12 N^{\circ} 1$ | ISSN: 1697-8293 | DOI: ri14.v12i1.650 

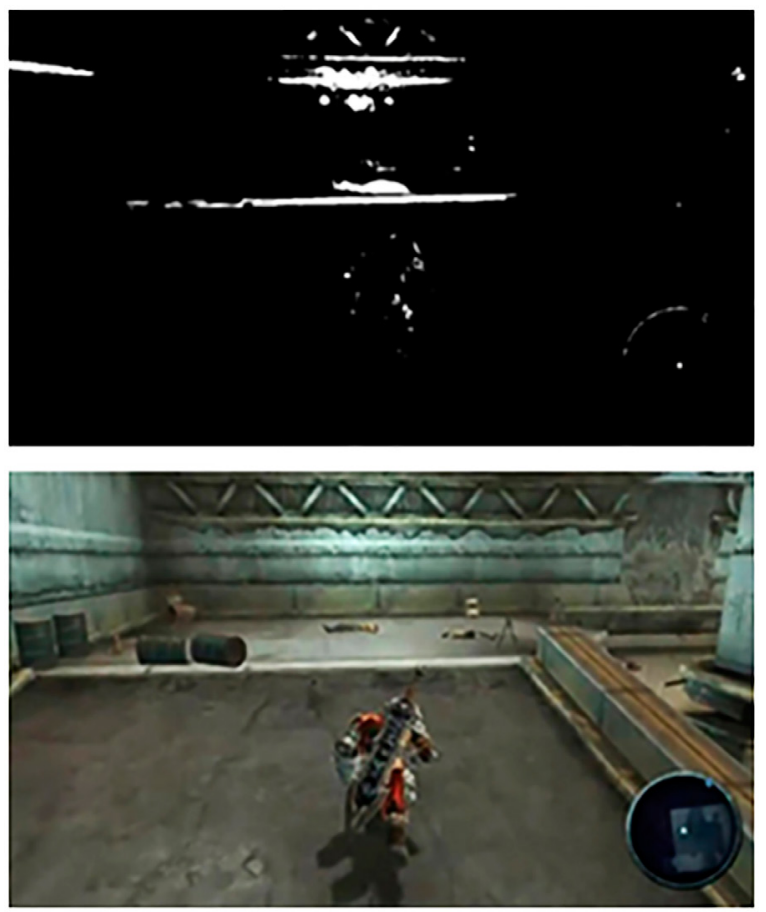

Figura 2: Las zonas blancas delimitadas por el Umbral muestran las partes de la imagen que mayor intensidad luminica muestran. Fuente: Darksiders (Vigil Games, 2010).

El contraste cromático es uno de los medios más poderosos para discriminar determinados objetos o elementos de una composición. Si este contraste se realiza además entre colores cálidos y fríos es posible ejercer un mayor nivel de atracción del observador hacia los elementos que presenten el color cálido. Los colores cálidos parecen hacernos una invitación, en tanto que los fríos nos mantienen a distancia (Arnheim, 1995).

Por último, las sombras en ocasiones oscurecen y limitan la visión, pero también pueden revelar detalles que apenas serían imperceptibles sin ellas, como las texturas (Brown, 2008). Entre las principales funciones de las sombras, destacan las de revelar ángulos alternativos (al mostrar, a modo de sombras proyectadas en la pared o en el suelo, formas del objeto que desde un único punto de vista no se pueden apreciar), añadir contraste y sugerir la presencia de objetos del fuera de campo.

DOI: ri14.v12i1.650 | ISSN: 1697-8293 | Año 2014 Volumen 12 N 1 | ICONO14 
Un caso muy representativo de esta última función se da en $M$, el vampiro de Düsseldorf (M, Fritz Lang, 1931), donde la aparición en cuadro del asesino tiene lugar mediante su sombra proyectada en la pared, la cual muestra un cartel que hace referencia a su búsqueda. En determinados videojuegos como Dead Space 2 se observa un empleo de sombras proyectadas para indicar al jugador que algo peligroso se encuentra cerca (Figura 3).

Frente a las sombras inherentes o atachadas, provocadas cuando las superficies que se encuentran en una dirección contraria hacia la que se dirige la fuente de luz reciben iluminación indirecta, las sombras proyectadas son aquellas que un objeto lanza hacia otra superficie mientras el primero se interpone en el camino que recorre la luz (Bordwell \& Thompson, 1995).
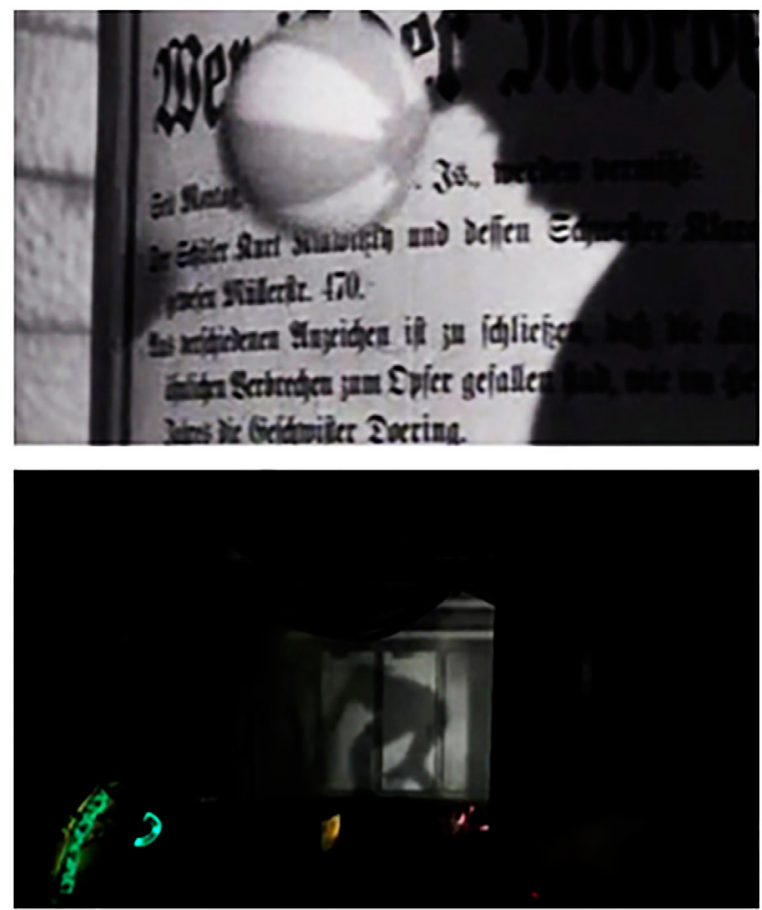

Figura 3: Empleo de las sombras proyectadas para sugerir al espectador/jugador lo que se encuentra en el fuera de campo. Fuente: $M$, el vampiro de Düsseldorf (M, Fritz Lang, 1931), Dead Space 2 (Visceral Games, 2011). 


\subsection{Constitución de categorías de análisis}

La investigación presente analiza el uso de la iluminación como forma de dar pistas sobre el movimiento que el jugador debe hacer por el espacio interactivo, por una parte, y sobre los objetos y non-player characters a los que tendrá acceso, por otra. Ante esta división, se trazaron dos categorías principales diferenciadas, basadas en el concepto de pistas de interacción. Este concepto fue empleado por Bardzell (2008) para designar aquellos signos presentes en la interfaz gráfica de usuario que comunican posibilidades de interacción al jugador. Las dos categorías de análisis de las que parte la investigación presente son las pistas de navegación y las pistas de interacción transmitidas a partir de la iluminación.

Bajo la categoría pistas de navegación se han registrado las ocasiones en las que se emplea la iluminación con la finalidad de condicionar el movimiento del jugador o de ayudarle a saber por dónde debe continuar.

Por otra parte, se han abordado bajo el término pista de interacción aquellas pistas destinadas a indicar al jugador qué non-player characters u objetos debe manipular para progresar en el juego.

\subsection{Tabla de análisis}

A partir del establecimiento de las categorías, se ha llevado a cabo un análisis de contenido en dos vertientes: una cualitativa y otra cuantitativa. Dado que el análisis de contenido cualitativo se basa en la lectura de un texto junto con un instrumento de recogida de información, se ha diseñado una tabla en la que registrar las observaciones realizadas (Figura 4). La tabla relaciona el uso de una o varias propiedades de la iluminación con la pregunta de la que provienen las categorías: ¿se emplea la luz para llamar la atención sobre un objeto o personaje con el que interactuar o para llamar la atención sobre un camino o un área que debe visitar? Asimismo, registra el momento en el que la pista de navegación o interacción fue detectada en el vídeo objeto de análisis, y proporciona una descripción del elemento que se muestra mediante la iluminación. 
La parte cuantitativa ha consistido en la contabilización del total de pistas de navegación detectadas, por una parte, y de las de interacción, por otra. Tras ello, se han elaborado porcentajes de las ocasiones en las que las diferentes propiedades de la iluminación son empleadas para mostrar pistas de navegación, por una parte, y las ocasiones en las que dichas propiedades se emplean para mostrar pistas de interacción, por otra.

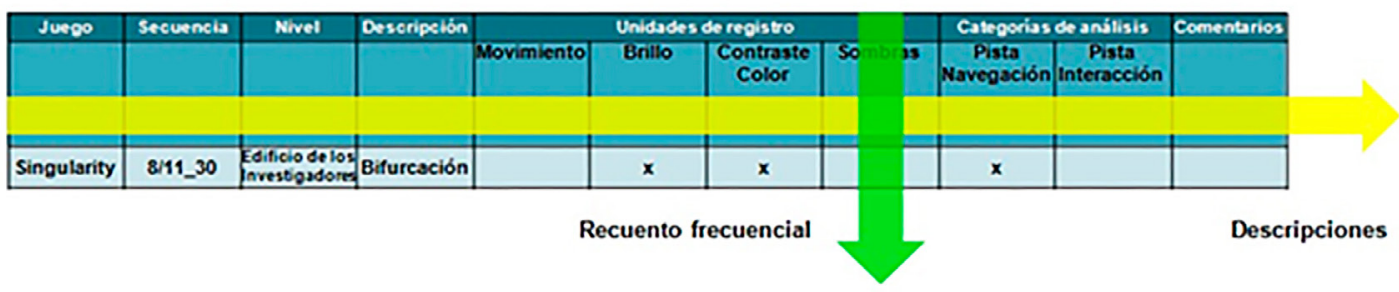

Figura 4. Modelo de tabla empleado para el análisis cualitativo y cuantitativo de la iluminación como herramienta para guiar la navegación y las interacciones del jugador

Los criterios para incluir en la tabla de análisis determinados usos de la iluminación han sido diferentes en lo referente a las pistas de interacción y a las pistas de navegación. Para las pistas de navegación, se ha contado la frecuencia con la que la iluminación (brillo, movimiento, sombras o contraste cromático) son empleadas para mostrar un camino. Con las pistas de interacción, en cambio, se ha procedido a omitir la contabilización de las veces en que se empleaba la iluminación para marcar objetos que aparecían con anterioridad en el mismo nivel. Determinados objetos, como las armas, se han contabilizado sólo la primera vez que aparecían en el nivel. La contabilización de cada una de las veces que se usa la iluminación para ello no resultaba práctico, dado que en que algunos casos, como las dagas de Castlevania: Lord of Shadows, son numerosas y presentan las mismas características.

\section{Resultados}

\subsection{Pistas de navegación}

De las propiedades de la iluminación tomadas en consideración para este análisis, ha sido la intensidad lumínica (sin acompañarse de ninguna otra propiedad)

ICONO14 | Año 2014 Volumen $12 N^{\circ} 1$ | ISSN: 1697-8293 | DOI: ri14.v12i1.650 
la que más se ha empleado en términos generales. Dos de los videojuegos pertenecientes a la muestra sólo usan esta propiedad para mostrar pistas de navegación. No obstante, se observa un uso significativo de la iluminación acompañada de movimiento (hasta el punto en el que algunos videojuegos lo usan para todas las pistas de navegación). Finalmente, las sombras se revelan como un elemento que no se usa para condicionar la navegación del jugador. La Figura 5 muestra las propiedades de la luz que se han identificado, así como las combinaciones de las mismas. También se proporciona el porcentaje de ocasiones en las que se han detectado con respecto al total de pistas de navegación obtenidas (106).

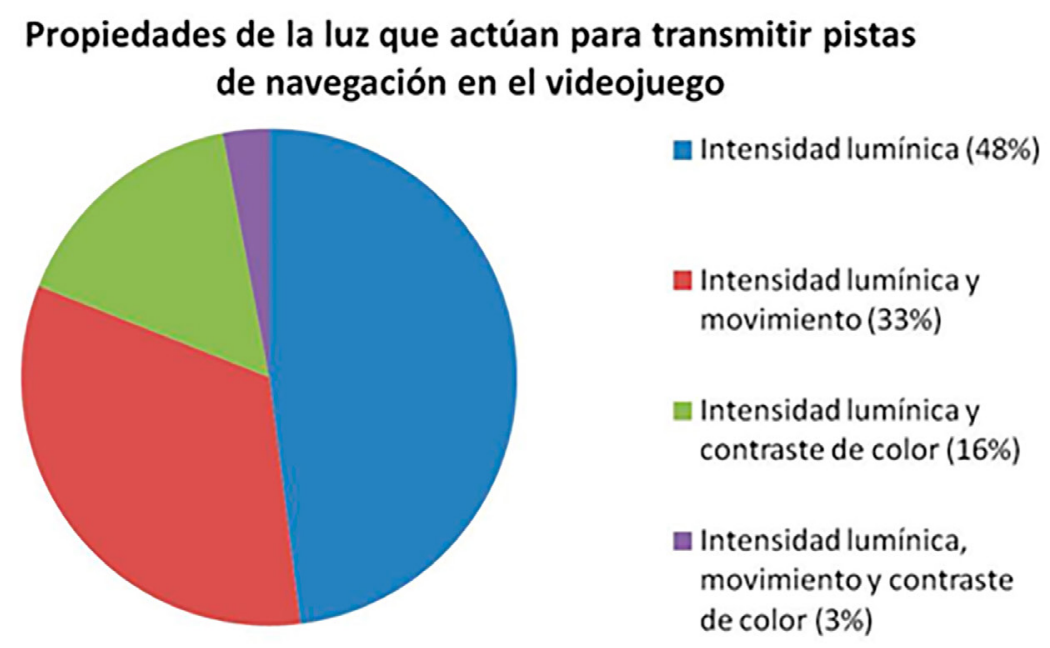

Figura 5. Propiedades de la luz empleadas como pista de navegación. Fuente: Elaboración propia.

La principal finalidad observada en el empleo de la iluminación para mostrar al jugador los caminos que debe tomar para proseguir su recorrido por el entorno interactivo (mediante brillo y brillo acompañado de movimiento principalmente, seguido del empleo de brillo con contraste cromático y brillo acompañado de movimiento y contraste cromático) parece ser la de permitir que el jugador se mueva fluidamente por el entorno y descartar tiempos muertos derivados de la búsqueda de la salida correcta. No obstante, se han observado pistas de navegación que, al tiempo que indican al jugador la dirección que debe tomar, ejercen una influencia importante en el desempeño de las misiones del juego. Es el caso de la persecución que tiene lugar en el nivel "Robo en el Museo" de Uncharted 3, donde el jugador

DOI: ri14.v12i1.650 | ISSN: 1697-8293 | Año 2014 Volumen 12 Nº 1 | ICONO14 
debe ser lo más rápido posible para evitar ser capturado por sus perseguidores (Figura 6).

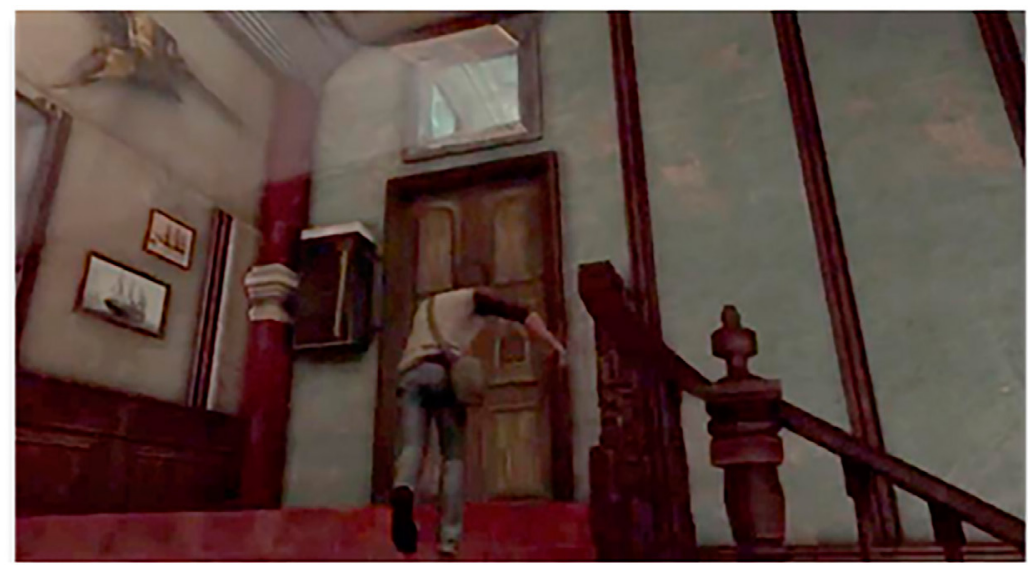

Figura 6: Ejemplo de intensidad lumínica aplicada a la salida que debe tomar el jugador. Ante una persecución, el jugador debe decidir rápido que salida tomar. La ventana es la única salida que permite el paso. Fuente: Uncharted 3 (Naughty Dog, 2011).

El tiempo en este juego se convierte en un factor determinante que condiciona la navegación del jugador, puesto que las reglas del juego determinan que la navegación rápida es condición indispensable para cumplir el objetivo impuesto (de lo contrario, será alcanzado por los enemigos). En este caso, el mayor nivel de intensidad lumínica de la ventana parece ser clave para que el jugador se dirija directamente a la misma y descarte la otra opción que se le presenta para huir: la puerta (que se encuentra bloqueada).

Además de ser clave para el desempeño correcto de algunas misiones, la iluminación parece ser una herramienta importante para atraer al jugador hacia determinadas áreas del entorno donde éste puede encontrar recursos u objetos necesarios para cumplir una misión (como en Bioshock 2 o Darksiders) o donde pueden tener lugar determinados eventos (como el enfrentamiento contra un personaje hostil en Dead Space 2). 


\subsection{Pistas de Interacción}

Las propiedades de la luz más empleadas para llamar la atención sobre los elementos con los que es necesario interactuar son el movimiento y el brillo. De manera menos frecuente, el contraste cromático y las sombras en movimiento, empleados principalmente para mostrar non-player characters. La Figura 7 muestra las propiedades de la luz que se han identificado, así como las combinaciones de las mismas. También se proporciona el porcentaje de ocasiones en las que se han detectado con respecto al total de pistas de interacción obtenidas (232).

Se observa un uso extendido de estas propiedades de la luz en pick ups (recursos de salud, armas, etc), objetos de misión, así como en puertas, ganchos y otros elementos con los que es necesario interactuar para avanzar por el espacio. También se emplean estas propiedades para revelar estrategias de juego en el enfrentamiento con enemigos (por ejemplo, un barril que presenta una intensidad lumínica elevada resulta ser un arma eficaz para vencer a una serie de personajes hostiles). El contraste cromático, si bien menos frecuente, se revela como una poderosa herramienta para delimitar visualmente lo que permite al jugador desempeñar una misión y lo que no, facilitando y optimizando la experiencia de juego. Es lo que se ha visto principalmente en Shadows of the Damned o en Bioshock 2. La luz cálida se emplea para aplicarla al objeto de importancia (Figura 8), mientras que la luz fría actúa como fondo y elemento de contraste. Sobre las sombras proyectadas, resulta significativo recalcar que éstas solo se usan para mostrar pistas de interacción.

Propiedades de la luz que actúan para transmitir pistas de interacción en el videojuego
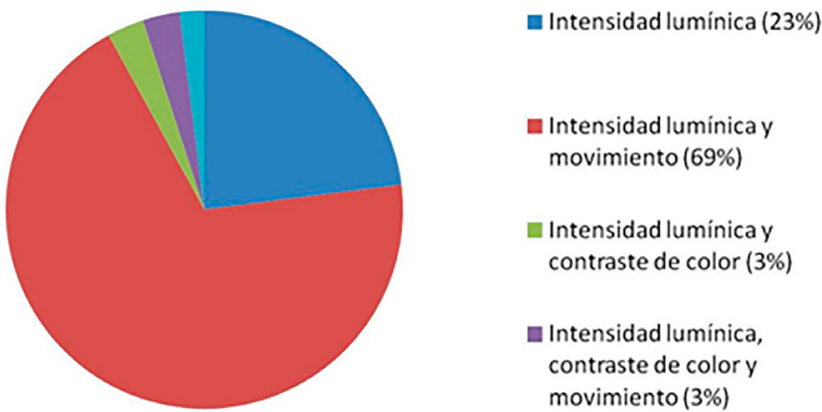

Figura 7. Propiedades de la luz empleadas como pista de interacción. Fuente: Elaboración propia. 


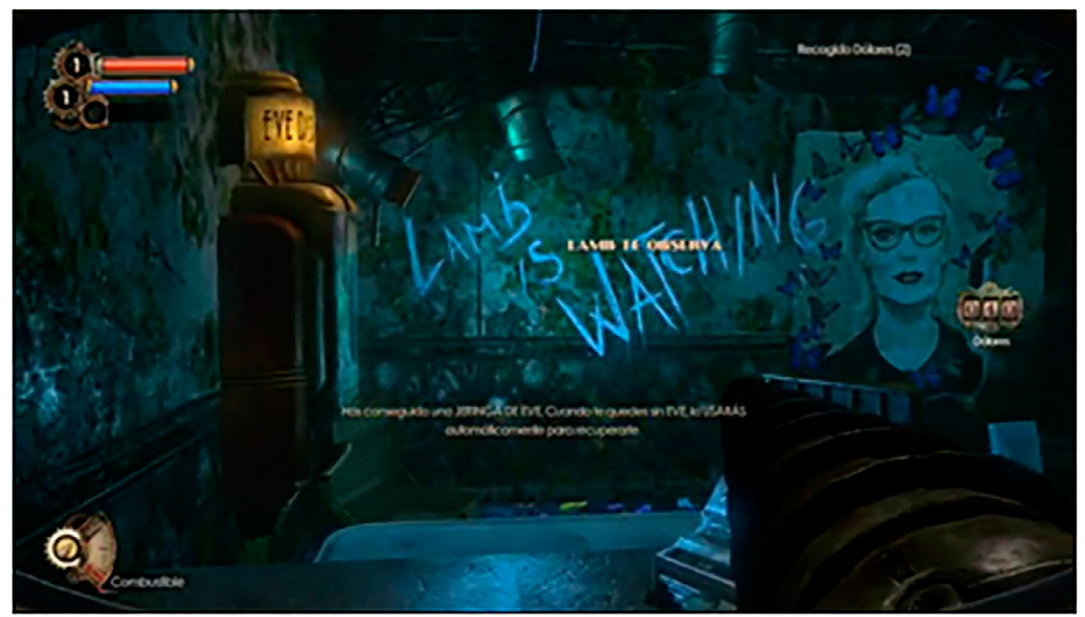

Figura 8. Empleo del contraste cromático para llamar la atención del jugador sobre un objeto interactivo. Fuente: Bioshock 2 (2K Games).

Algunas sombras provenientes de la iluminación dinámica, como las de los enemigos en Crysis 2 (Figura 9) pueden ser una mera consecuencia de la tecnología empleada y no una intención explícita del diseñador. Desde esta investigación se cree que, intencionalmente o no, pueden ser un elemento interesante para crear experiencias de juego.

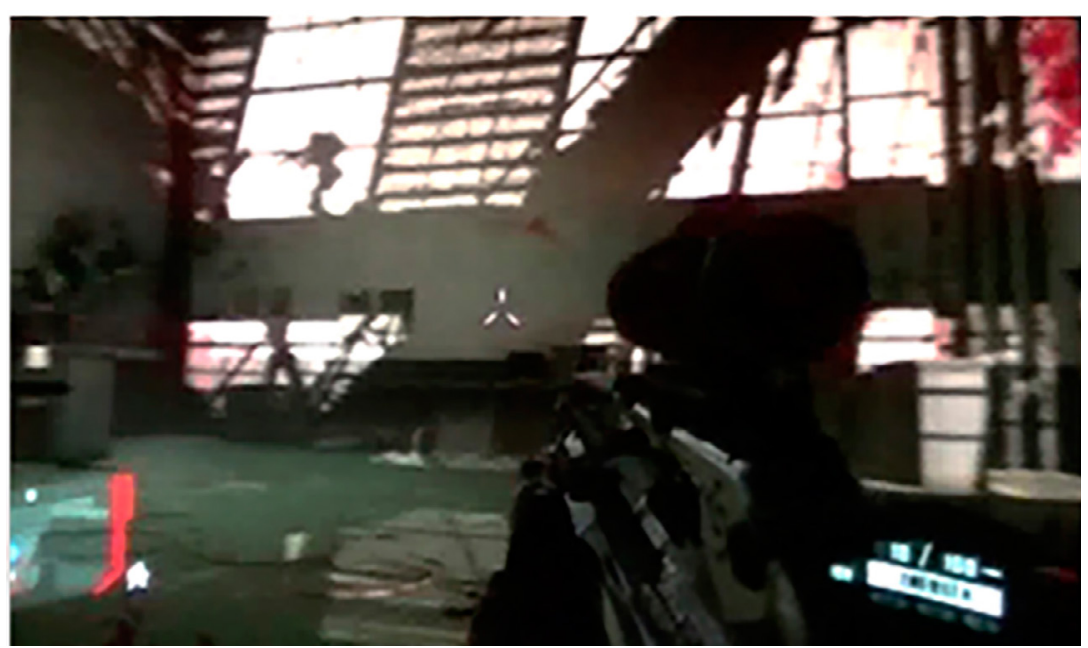

Figura 9. Empleo de las sombras proyectadas para advertir al jugador de la presencia de enemigos. Fuente: Crysis 2 (Crytek). 
En ocasiones el encuentro del jugador con el personaje que proyecta la sombra es instantáneo (Dead Space 2). En otros parece que se juega con las expectativas del jugador, como es el caso de Bioshock 2 en el nivel "Expreso Atlántico", donde la sombra del enemigo no da lugar a que el enemigo aparezca inmediatamente. En lugar de ello, se crean unos momentos de incertidumbre antes de que el jugador se encuentre con el enemigo, que aparece desde un ángulo inesperado. Las sombras proponen una interacción, pero lo hacen de una manera sugerente, lo que se aleja de las pistas de interacción en otras aplicaciones digitales de calado más productivo y menos orientado al disfrute como los videojuegos.

\section{Discusión}

Los diseñadores de niveles son partícipes de la responsabilidad de crear experiencias de juego a través de la construcción de espacios sobre los que el jugador realiza sus interacciones. En este sentido, la iluminación, además de influir en el estado emocional del jugador (Knez \& Niedenthal, 2008), y de ser una poderosa herramienta para evocar atmósferas (Niedenthal, 2008), se ha convertido en un recurso para apoyar la navegación y las interacciones del jugador, tal y como se muestra en el análisis realizado. Dada la tendencia del ser humano a elegir los caminos de mayor intensidad lumínica (Taylor \& Socov, 1974), las fuentes de luz en los entornos tridimensionales lúdicos parecen disponerse de tal modo que permitan captar la atención del jugador sobre los ítems con los que debe interactuar para progresar en el juego.

Esto, unido a los experimentos de Seif El-Nasr y Yan (2006), que confirman que el establecimiento de focos visuales de atención favorecen las interacciones del jugador y su progreso en el juego, permite considerar que la iluminación es susceptible de ser empleada para generar experiencias de juego satisfactorias, al eliminar la frustración derivada de no encontrar los ítems y las áreas del entorno que van a permitir el progreso en el juego, al facilitar el desempeño de misiones o propiciar determinados eventos en los juegos, y al permitir que determinados comportamientos se produzcan con más probabilidad que otros. 
A pesar de estas consideraciones, el método empleado en el análisis de este trabajo no trata de hacer una predicción exacta del comportamiento de los jugadores a partir de las características de la iluminación, sino proporcionar una forma de diseñar niveles en base a la experiencia que se espera o se desea generar en el jugador, en términos de lo que Tychsen y Canossa (2008) llaman play persona. No obstante, es necesario añadir que la experiencia de juego no sólo depende de los elementos propios del juego (objeto de análisis en este trabajo), sino de características del jugador tales como el contexto sociocultural en el que se encuentra y sus experiencias previas (Ermi \& Mäyrä, 2005).

\section{Conclusión}

Este trabajo surgía de la idea de que la estética en el videojuego, más allá de limitarse al estudio de los estilos visuales o a la calidad de los gráficos (aspectos que se suelen valorar negativamente, al considerarse que es más importante la calidad del juego que la de la representación gráfica del mismo) puede ser entendida como un fenómeno sensorial y perceptual, que actúa como un instrumento o perspectiva desde la que estudiar las cualidades de la experiencia de juego. En este contexto, la investigación se centraba en un recurso expresivo escasamente explorado en el videojuego: la iluminación. Se partía del supuesto de que este elemento visual es empleado como instrumento para configurar la experiencia interactiva del jugador en los entornos de los videojuegos tridimensionales.

La iluminación, a través de cuatro propiedades fundamentales de la luz (el movimiento, la intensidad lumínica, el contraste de color y las sombras proyectadas), se aplica para apoyar las interacciones y la navegación del jugador en los entornos tridimensionales lúdicos. Gran parte de estas interacciones no sólo tienen que ver con averiguar un camino para eliminar tiempos muertos 0 coger objetos e interactuar con personajes, sino que se encuentran integradas en las mecánicas de juego y la gameplay.

Las propiedades de la luz más empleadas para transmitir pistas de navegación han sido la intensidad lumínica y el movimiento. Las sombras proyectadas,

ICONO14 | Año 2014 Volumen $12 N^{\circ} 1$ | ISSN: 1697-8293 | DOI: ri14.v12i1.650 
en cambio, no se han detectado en ningún caso. La luz se aplica principalmente para mostrar elementos del espacio que son significativos para el avance del jugador en el juego. Si bien la investigación partía de la idea de que las pistas de navegación se transmitían con el fin de no causar tiempos muertos ni producir un sentimiento de desorientación en el jugador, se ha visto que la iluminación como modo de guiar el movimiento del jugador tiene un alcance mayor en la experiencia de juego. Las ayudas a la navegación transmitidas mediante este recurso visual, además de proporcionarse para orientar espacialmente al jugador, se proporcionan para optimizar el movimiento del jugador en base a las actividades lúdicas prediseñadas en el juego.

En lo relativo a pistas de interacción, las propiedades de la luz que con mayor frecuencia se han detectado han sido la intensidad lumínica acompañada de movimiento $\mathrm{y}$, en menor medida, la intensidad lumínica.

En la mayoría de los casos detectados las pistas de interacción se dan de forma directa, pero en casos aislados se ha observado que la luz se emplea para crear incertidumbre y provocar cierto sentimiento de desorientación o de sorpresa en el jugador. Este tipo de usos de los recursos visuales expresivos pone de relieve el carácter especial del videojuego a la hora de ser analizado bajo la perspectiva de la usabilidad. En determinadas ocasiones la confusión y la dificultad en el videojuego forman parte de la experiencia interactiva global.

La investigación presente se ha centrado en el estudio del videojuego, no del jugador. Si bien a partir de teorías relativas a la atención visual, al diseño de la interacción y al diseño de videojuegos se han identificado una serie de usos de la iluminación destinados a optimizar la navegación y las interacciones del usuario en los videojuegos, este trabajo es un estudio formalista centrado en el juego. Es por ello por lo que una investigación experimental, a partir de prototipos, que relacione los resultados de esta investigación con el comportamiento real del jugador, supondría una gran aportación al diseño de videojuegos y, en especial, al diseño de niveles, al tiempo que aportaría una valiosa información en lo relativo a experiencias de juego. 
Por otra parte, esta investigación ha realizado una aproximación a los videojuegos comerciales o mainstream de los géneros first person shooter y acción-aventura. En un futuro se pretende tomar en consideración las propuestas de videojuegos independientes $\mathrm{y} / \mathrm{o}$ pertenecientes a otros géneros, que podrían aportar nuevas ideas y perspectivas desde las que abordar la iluminación.

\section{Notas}

[1] http://www.metacritic.com/

\section{Referencias}

Arnheim, R (1995). Arte y Percepción Visual. Madrid: Alianza.

Bardin, L (1996). El análisis de contenido. Madrid: Akal.

Bardzell, S (2008). Systems of Signs and Affordances: Interaction Cues in

3D Games. En Leino, 0.; Wirman, H. y Fernández, A. (Eds), Extending

Experiences. Structure, analysis and design of computer game player

experience, (pp.191 - 209). Rovaniemi: Lapland University Press.

Bechtel, R (1967), Human movement and architecture. Trans-action, 4 (6), 53-

56.

Bordwell, D \& Thompson, K (1995). El arte cinematográfico. Barcelona: Paidós.

Brown, B (2008). Motion Picture and Video Lighting. Boston: Focal Press.

Canossa, A (2006). Designing Levels for Enhanced Player Experience Mental

tools for gameworld designers. I0 Interactive / Denmark's School of Design.

Recuperado de http://www.itu.dk/people/alec/Enhanced\%20Player\%20

Experience01.pdf

Cooper, A., Reimann, R. \& Cronin, D (2007). About Face 3. The Essentials of Interaction Design. Indianapolis: Wiley.

Ermi, L. \& Mäyrä, F (2005), Fundamental Components of Gameplay Experience: Analysing Immersion. En de Castell, S. y Jenson, J. (Eds), Changing Views: Worlds in Play. Selected Papers of the 2005 Digital Games Research 
Association's Second International Conference (pp. 15-27). Recuperado de http://people.uta.fi/ tlilma/gameplay_experience.pdf

Isbister, K. \& Schaffer, N (2008). Game Usability: Advancing the Player Experience. Burlington: Morgan Kaufmann.

Juul, J (2002). The 0pen and the Closed: Game of emergence and games of progression. En Computer Games and Digital Cultures Conference Proceedings, 323 - 329. Tampere: Tampere University Press. Recuperado de http://www. jesperjuul.net/text/openandtheclosed.html

Knez, I. \& Niedenthal, S. (2008). Lighting in digital game worlds: effects on affect and play performance. CyberPsychology and Behavior, 11(2), 129-133. Michel, L. 1996. Light: the shape of space. Designing with space and light. Nueva York: Wiley.

Milam, D. and Seif El-Nasr, M (2010a). Design Patterns to Guide Player Movement in 3D Games. En Sandbox '10 Proceedings of the 5th ACM SIGGRAPH Symposium on Video Games (pp. 37 - 42). Nueva York: ACM. DOI= http://doi. acm.org/10.1145/1836135.1836141

Milam, D. y Seif El-Nasr, M (2010b). Analysis of level design 'push \& pull' within 21 games. En Proceedings of the Fifth International Conference on the Foundations of Digital Games (pp. 139 - 146). Nueva York: ACM. DOI= http:// doi.acm.org/10.1145/1822348.1822367

Niedenthal, (2008). Complicated shadows: The aesthetic significance of simulated illumination in digital games. Tesis doctoral. Universidad de Malmö, School of Arts and Communication. Malmö.

Nitsche, M (2008). Video Game Spaces: Image, Play, and Structure in 3D Worlds. Cambridge/Londres: The MIT Press.

Seif El-Nasr, M., Vasilakos, T., Rao, C. y Zupko, J (2009). Dynamic Intelligent Lighting for Directing Visual Attention in Interactive 3D Scenes. IEEE Transactions on Computational Intelligence and AI in Games, 1(2), 145 - 153. Seif El-Nasr, M. y Yan, S (2006). Visual Attention in 3D Video Games. En ACE '06 Proceedings of the 2006 ACM SIGCHI international conference on Advances in computer entertainment technology. Nueva York: ACM. DOI= http://doi.acm. org/10.1145/1178823.1178849

Sennerster, C. (2008). Gameplay (3D Game Engine + Ray Tracing = Visual Attention through Eye Tracking). Tesis doctoral. Universidad de Malmö, School 
of Arts and Communication. Malmö.

Taylor, L. H. y Socov, E.W (1974). The movement of people towards lights.

Journal of Illuminating Engineering Society, 3, 237-241.

Tychsen, A. y Canossa, A (2008). Defining personas in games using metrics. En

Proceedings of the 2008 Conference on Future Play: Research, Play, Share (pp. 73 - 80). Nueva York: ACM.

DOI= http://doi.acm.org/10.1145/1496984.1496997 